\title{
A STRUCTURE THEOREM FOR A PAIR OF QUADRATIC FORMS
}

\author{
J. S. HSIA, M. JÖCHNER, AND Y. Y. SHAO
}

(Communicated by William W. Adams)

\begin{abstract}
Dedicated to Professor Zhu Fu-Zu on the occasion of his seventy-fifth birthday
\end{abstract}
\begin{abstract}
For any two lattices $L$ and $K$ in the same genus there exist isometric primitive sublattices $L^{\prime}, K^{\prime}$ of codimension 1 . This result not only proves Friedland's conjecture but also extends it to lattices in an arbitrary genus and defined over any algebraic number field.
\end{abstract}

In [F] Friedland proved that for any two positive definite integral symmetric matrices $A$ and $B$ of rank $n$ and determinant unity there are matrices $A_{1}=\left(a_{i j}\right)$ and $B_{1}=\left(b_{i j}\right)$ which are $\mathbb{Z}$-equivalent (as quadratic forms) to $A$ and $B$ respectively and such that $a_{i j}=b_{i j}$ for $1 \leq i, j \leq n-2$. He further conjectured that the same holds with $1 \leq i, j \leq n-1$. His proof is matricial. We shall give here not only a lattice-theoretic proof of his theorem, but also a more general result not restricted to unimodular lattices rather for any genus and defined over any number field. Furthermore, we shall also prove the conjectured codimension-one result in this full general setting. In the course of the proof we shall first present a weaker version (Proposition) which is based on the theorem of "spinor linkage" $[\mathrm{BH}]$. This version is weaker because the constructed pair of isometric sublattices in general has codimension two and will only have codimension one when the given lattices corresponding to the matrices $A$ and $B$ belong to the same spinor genus. However, this version has the virtue that the isometric sublattices are more effectively determinable since the effectiveness of finding the prime spot in the construction of the associated graph had been demonstrated in [BH]. The more general statement in the structure theorem below uses the stronger property of "class linkage" which is of independent interest.

In what follows, let $F$ be an algebraic number field, $R$ its ring of integers, and $V$ a regular quadratic space over $F$ of dimension $n$. Let $L$ and $K$ be two $R$-lattices on $V$ belonging to the same genus. $L$ and $K$ are said to be $q$-neighbors (for some discrete spot $q$ on $F$ ) if $L_{p}=K_{p}$ for $p \neq q$ and $\left[L_{q}: L_{q} \cap K_{q}\right]=\left[K_{q}: L_{q} \cap K_{q}\right]=\operatorname{Norm}(q)$. The "spinor genera linkage theorem" of $[\mathrm{BH}]$ states that we can always effectively find a discrete spot $q$ away from $2 \operatorname{vol}(L)$ and a lattice $K^{\prime}$ belonging to $\operatorname{spn}^{+} K$ so that $L$ and $K^{\prime}$ are

Received by the editors September 3, 1991 and, in revised form, March 23, 1992.

1991 Mathematics Subject Classification. Primary 11E12; Secondary 15A63, 11D09, 11 E20.

Research partially supported by N.S.F. 
$q$-neighbors. Moreover, there is an $R_{q}$-basis $e_{1}, f_{1}, \ldots, e_{s}, f_{s} ; z_{2 s+1}, \ldots, z_{n}$ for $L_{q}$ where the $e_{j}$ and $f_{j}$ are mutually orthogonal hyperbolic pairs and $s$ is the Witt index of $V_{q}$, and

$$
K_{q}^{\prime}=\left(R_{q} q^{-1} e_{1}+R_{q} q f_{1}\right) \perp\left(R_{q} e_{2}+R_{q} f_{2}+\cdots+R_{q} z_{n}\right) .
$$

We set $M_{(q)}=R_{q}\left(e_{1}+q f_{1}\right) \perp\left(R_{q} e_{2}+R_{q} f_{2}+\cdots+R_{q} z_{n}\right)$. It is clear that $M_{(q)}$ is a primitive sublattice of codimension 1 in both $L_{q}$ and $K_{q}^{\prime}$. By the Chinese Remainder Theorem, take a global sublattice $J$ of $L \cap K^{\prime}$ of codimension 1 which is $q$-adically close to $M_{(q)}$, and suppose $J$ lies on the subspace $U$. We construct a sublattice $X$ by $X_{q}=J_{q}$ and $X_{p}=U_{p} \cap L_{p}$ for all $p \neq q$. Clearly, $X$ is a primitive sublattice of codimension 1 in both $L$ and $K^{\prime}$.

Suppose $V$ is indefinite and $\operatorname{dim} V \geq 3$. Then it is well known that $\operatorname{spn}^{+} K$ $=\operatorname{cls}^{+} K$ so that we have isometric primitive sublattices $X_{L}$ and $X_{K}$, each of codimension 1, of $L$ and $K$ respectively.

Next suppose $V$ is a definite (i.e., totally definite) space, say it is positive definite at a certain infinite spot and has $\operatorname{dim} V \geq 2$. We make an orthogonal adjunction $\widetilde{V}:=V \perp[-1]$; similarly, $\widetilde{L}:=L \perp\langle-1\rangle$ and $\widetilde{K}:=K \perp\langle-1\rangle$. It is convenient to introduce the notation $\subseteq^{*}$ to denote primitive containment and $\subseteq^{*}{ }_{t}$ to denote the primitive containment with codimension of at most $t$. We first need some simple observations:

(i) If $A \subseteq{ }^{*}{ }_{t} B \subseteq^{*}{ }_{s} C$, then $A \subseteq{ }^{*}{ }_{t+s} C$;

(ii) If $A \subseteq^{*}{ }_{t} C$ and $B \subseteq^{*} C$, then $A \cap B \subseteq^{*} B$.

Only part (ii) perhaps requires some explanations. For this it is sufficient to show that $F(A \cap B) \cap B \subseteq A \cap B$. But we have $F(A \cap B) \cap B \subseteq F A \cap F B \cap B=$ $F A \cap B=F A \cap C \cap B=A \cap B$.

By the above, we have isometric primitive codim 1 sublattices $\widetilde{X} \subseteq^{*} \widetilde{L}$, and $\widetilde{Y} \subseteq \widetilde{K}$. Set $X=\widetilde{X} \cap L$. By (ii) we have $X \subseteq^{*}{ }_{1} L$ and also $X \subseteq^{*}{ }_{1} \widetilde{X}$. Let $\sigma$ be an isometry from $\widetilde{X}$ to $\widetilde{Y}$. Then $\sigma X \subseteq^{*}{ }_{1} \widetilde{Y}$. So by (i) and (ii) $\sigma X \subseteq^{*}{ }_{2} \widetilde{K}$, hence $\sigma X \cap K \subseteq^{*}{ }_{2} K$ and $\sigma X \cap K \subseteq^{*} \widetilde{Y}$. Applying $\sigma^{-1}$ to the last containment, we have $X \cap \sigma^{-1} K \subseteq^{*} \widetilde{X}$. Hence, $X \cap \sigma^{-1} K \subseteq^{*} L$ by (i) and (ii). This gives primitive isometric sublattices $X \cap \sigma^{-1} K \subseteq^{*} L$ and $\sigma X \cap K \subseteq^{*} K$ of codimension at most 2.

Finally, suppose $L$ belongs to the spinor genus of $K$. Then, $\widetilde{L}$ and $\widetilde{K}$ belong to the same proper class. We can then choose $\widetilde{X}=\widetilde{L}$ and $\widetilde{Y}=\widetilde{K}$ and repeat the same procedure to conclude the existence of isometric primitive codimension 1 sublattices in $L$ and $K$.

Summarizing, we have the following result:

Proposition. Let $L$ and $K$ be two $R$-lattices on $V$ belonging to the same genus. Then there exist isometric primitive sublattices $X \subseteq^{*} L$ and $Y \subseteq^{*} K$ of codimension

(i) 1 if $V$ is not totally definite, and $\operatorname{dim} V \geq 3$;

(ii) 2 if $V$ is totally definite, and $\operatorname{dim} V \geq 2$;

(iii) 1 if $V$ is totally definite and $L \in \operatorname{spn} K$ and $\operatorname{dim} V \geq 2$.

Next, we prove the key lemma which permits the improvement of the proposition to the theorem below. The proof of this lemma depends on the number field version of Lemma 1.6 of [HKK], which we shall also refer to by the same 
reference. It should also be remarked that this approximation Lemma 1.6 does not hold if the ambient space is a hyperbolic plane.

Lemma ("Class Linkage"). Let $L$ and $K$ be two $R$-lattices in the same genus on a regular quadratic space $V$ of $\operatorname{dim} V \geq 2$, and we suppose that $V$ is not a hyperbolic plane. Then, there is an $L^{\prime}$ in the proper class of $L$ and a prime spot $q$ such that $L^{\prime}$ and $K$ are q-neighbors.

Proof. Let $S$ be a finite set of primes containing all dyadic ones and such that when $p$ is not in $S$ then $L_{p}=K_{p}=$ unimodular. For $p \in S$, let $\sigma_{p} \in O^{+}\left(V_{p}\right)$ with $\sigma_{p}\left(L_{p}\right)=K_{p}$. Without loss of generality, we may write $\sigma_{p}=\tau_{x_{1}^{p}} \cdots \tau_{x_{m}^{p}}$ for all $p \in S$ with fixed $m$. Here $x_{i}^{p}$ is in $L_{p}$ and $\tau_{x_{i}^{p}}$ is the symmetry associated with $x_{i}^{p}$. By Lemma 1.6 of [HKK], there is $x_{m} \in L$ and a prime $q_{m} \notin S$ satisfying that $x_{m}$ is close to $x_{m}^{p}$ at all prime $p \in S$; and when $q \notin S \cup\left\{q_{m}\right\}$, $\operatorname{ord}_{q}\left(Q\left(x_{m}\right)\right)=0$ and $\operatorname{ord}_{q_{m}}\left(Q\left(x_{m}\right)\right)=1$.

Suppose we have already constructed the vectors $\left\{x_{i}, \ldots, x_{m}\right\}$ and the corresponding primes $\left\{q_{i}, \ldots, q_{m}\right\}$ with $1<i \leq m$ having the properties $(\star)$ :

for each $j$ with $i \leq j<m-1$

$x_{j}$ is close to $x_{j}^{p}$ at all $p \in S$,

$x_{j}$ is close to $x_{j+1}$ at $q_{j+1}$,

$\operatorname{ord}_{p}\left(Q\left(x_{j}\right)\right)=0$ for all $p \notin S \cup\left\{q_{j}, q_{j+1}\right\}$, and

$\operatorname{ord}_{q_{j}}\left(Q\left(x_{j}\right)\right)=1$.

Now by Lemma 1.6 of [HKK] again, we have $x_{i-1} \in L$ and a prime $q_{i-1} \notin$ $S \cup\left\{q_{i}, \ldots, q_{m}\right\}$ such that $x_{i-1}$ is close to $x_{i-1}^{p}$ at all prime $p \in S ; x_{i-1}$ is close to $x_{i}$ at $q_{i} ; \operatorname{ord}_{q}\left(Q\left(x_{i-1}\right)=0\right.$ for all $q \notin S \cup\left\{q_{i-1}, q_{i}\right\}$; and $\operatorname{ord}_{q_{i-1}}\left(Q\left(x_{i-1}\right)\right)=1$. Note that the new sets of $x s$ and $q s$ still have the properties $(\star)$. Repeating this construction we get the sets $\left\{x_{1}, \ldots, x_{m}\right\}$ and $\left\{q_{1}, \ldots, q_{m}\right\}$ having properties $(\star)$.

Putting $\sigma=\tau_{x_{1}} \cdots \tau_{x_{m}}$, we see that $L^{\prime}=\sigma(L)$ is the desired lattice. For, if $p \in S$, then $\sigma$ is close to $\sigma_{p}$ and hence $\sigma\left(L_{p}\right)=\sigma_{p}\left(L_{p}\right)=K_{p}$. If $p \notin$ $S \cup\left\{q_{1}, \ldots, q_{m}\right\}$, then all $Q\left(x_{i}\right)$ are units so $\sigma\left(L_{p}\right)=L_{p}=K_{p}$. At $q_{i}$ with $1<i \leq m$, we write the $\sigma$ as

$$
\sigma=\left(\tau_{x_{1}} \cdots \tau_{x_{i-2}}\right)\left(\tau_{x_{i-1}} \tau_{x_{i}}\right)\left(\tau_{x_{i+1}} \cdots \tau_{x_{m}}\right) .
$$

By the condition $(\star)$ we see all three components of $\sigma$ are in $O\left(L_{p}\right)$, so we still have $\sigma\left(L_{p}\right)=L_{p}=K_{p}$. Finally let us check at $q_{1}$, because all $\tau_{x_{i}}$ with $i \geq 2$ are in $O\left(L_{p}\right)$; we have $\sigma\left(L_{q_{1}}\right)=\tau_{x_{1}}\left(L_{q_{1}}\right)$. Noting that $x_{1} \in L_{q_{1}}$ with $\operatorname{ord}_{q_{1}}\left(Q\left(x_{1}\right)\right)=1$, we can conclude that $\left[L_{q_{1}}: L_{q_{1}} \cap \sigma\left(L_{q_{1}}\right)\right]=\left[\sigma\left(L_{q_{1}}\right): L_{q_{1}} \cap\right.$ $\left.\sigma\left(L_{q_{1}}\right)\right]=\operatorname{Norm}\left(q_{1}\right)$. So $L^{\prime}=\sigma(L)$ and $K$ are $q_{1}$-neighbors.

The proof of the theorem is now exactly the same as that of part (i) of the proposition. The only change needed is to use the class linkage lemma instead of the spinor linkage theorem. Thus, we have:

Theorem. Let $L$ and $K$ be two $R$-lattices in the same genus on $V$ and $\operatorname{dim} V \geq$ 2. Assume that $V$ is not a hyperbolic plane. Then there exist isometric primitive sublattices $X \subseteq^{*} L$ and $Y \subseteq^{*} K$ of codimension 1 . 
Remark. It is not even necessary that $L$ and $K$ belong to the same genus. From the proof given it is clear that all that is required is that $\widetilde{L}$ and $\widetilde{K}$ belong to the same genus.

\section{REFERENCES}

[BH] J. W. Benham and J. S. Hsia, Spinor equivalence of quadratic forms, J. Number Theory 17 (1983), 337-342.

[F] S. Friedland, Normal forms for definite integer unimodular quadratic forms, Proc. Amer. Math. Soc. 106 (1989), 917-921.

[HKK] J. S. Hsia, Y. Kitaoka, and M. Kneser, Representations of positive definite quadratic forms, J. Reine Angew. Math. 301 (1978), 132-141.

(J. S. Hsia and Y. Y. Shao) Department of Mathematics, Ohio State University, ColumBUS, Оніо 43210

E-mail address, J. S. Hsia: jshsia@math.ohio-state.edu

E-mail address, Y. Y. Shao: shao@math.ohio-state.edu

(M. Jöchner) Department of Mathematics, University of Southern California, Los ANGELES, CALIFORNIA 90089

Current address: Kanalstrasse 25, 7022 L-Echterdingen, Germany

E-mail address: 100116.3445@compuserve.com 